\title{
Formononetin, a methoxy isoflavone, enhances bone regeneration in a mouse model of cortical bone defect
}

\author{
Krishna Bhan Singh ${ }^{1}$, Manisha Dixit ${ }^{1}$, Kapil Dev ${ }^{2}$, Rakesh Maurya ${ }^{2}$ and Divya Singh ${ }^{1 *}$ \\ ${ }^{1}$ Division of Endocrinology, Central Drug Research Institute (Council of Scientific and Industrial Research), Sector 10, \\ Jankipuram Extension, Sitapur Road, Lucknow 226031, Uttar Pradesh, India \\ ${ }^{2}$ Medicinal \& Process Chemistry Division, Central Drug Research Institute (Council of Scientific and Industrial Research), \\ Sector 10, Jankipuram Extension, Sitapur Road, Lucknow 226031, Uttar Pradesh, India
}

(Submitted 24 February 2017 - Final revision received 24 May 2017 - Accepted 27 May 2017)

\section{Abstract}

The bone regeneration and healing effect of formononetin was evaluated in a cortical bone defect model that predominantly heals by intramembranous ossification. For this study, female Balb/c mice were ovariectomised (OVx) and a drill-hole injury was generated in the midfemoral bones of all animals. Treatment with formononetin commenced the day after and continued for $21 \mathrm{~d}$. Parathyroid hormone $\left(\mathrm{PTH}_{1-34}\right)$ was used as a reference standard. Animals were killed at days 10 and 21. Femur bones were collected at the injury site for histomorphometry studies using microcomputed tomography $(\mu \mathrm{CT})$ and confocal microscopy. RNA and protein were harvested from the region surrounding the drill-hole injury. For immunohistochemistry, $5 \mu \mathrm{m}$ sections of decalcified femur bone adjoining the drill-hole site were cut. $\mu$ CT analysis showed that formononetin promoted bone healing at days 10 and 21 and the healing effect observed was significantly better than in Ovx mice and equal to PTH treatment in many aspects. Formononetin also significantly enhanced bone regeneration as assessed by calcein-labelling studies. In addition, formononetin enhanced the expression of osteogenic markers at the injury site in a manner similar to PTH. Formononetin treatment also led to predominant runt-related transcription factor 2 and osteocalcin localisation at the injury site. These results support the potential of formononetin to be a bone-healing agent and are suggestive of its promising role in the fracture-repair process.

Key words: Osteoporosis: Bone healing: Bone regeneration: Microcomputed tomography: Formononetin: Parathyroid hormone

According to the global status report on road safety by the WHO, India has the worst traffic-accident rates worldwide with over 130000 deaths annually. Many of these accidents lead to fractures of delayed-union or non-union type, which can result in multiple surgeries and cause significant patient morbidity and loss of limb function. Apart from accidental fractures, disorders associated with systemic inflammation such as diabetes mellitus and rheumatoid arthritis can prolong or disturb fracture healing ${ }^{(1)}$.

Studies also link osteoporosis to systemic inflammation, and fracture healing has been shown to be decelerated in osteoporosis ${ }^{(2)}$. With the increasing burden of trauma, identifying therapeutics for enhancing fracture healing remains paramount. Our interest is in the administration of stimulating factors to accelerate bone healing. Although the Food and Drug Administration has approved recombinant human bone morphogenetic protein 2 (BMP2), marketed as INFUSE $^{\circledR}$ Bone Graft, for treating open tibial-shaft fractures, it is very expensive and associated with many side effects including postoperative inflammation, cyst-like bone formation and life-threatening cervical swelling ${ }^{(3)}$. Besides recombinant BMP2, agents like parathyroid hormone (PTH) have been tested for their ability to heal fractures. Daily systemic injections of PTH have been shown to enhance fracture healing in several rodent models ${ }^{(4,5,6)}$. The disadvantage with PTH is again its very high cost and the route of administration ${ }^{(7,8)}$. Thus, the need for agents that promote bone healing and regeneration is a worldwide challenge.

Studies in our laboratory showed that Butea monosperma plant extract, enriched in isoflavones, has potent osteogenic activity $^{(9)}$. It was observed that compounds majorly contributing to the bone-conserving effect of Butea extract included methoxy isoflavones like isoformononetin, formononetin, cajanin, cladrin and a natural pterocarpan: medicarpin. Among these, formononetin (7-hydroxy-3(4-methoxyphenyl) chromone or 4'-methoxy daidzein), a soya isoflavonoid, was found abundantly in $B$. monosperma ${ }^{(10)}$. Formononetin is found in a number of plants and herbs like red clover ${ }^{(11)}$. It predominantly occurs in leguminous plants and Fabaceae,

Abbreviations: $B M P 2$, bone morphogenetic protein 2; BV/TV, trabecular bone volume; DA, degree of anisotropy; DAPI, 4',6-diamidino-2-phenylindole; OCN, osteocalcin; OVx, ovariectomised; PTH, parathyroid hormone; RUNX2, runt-related transcription factor 2; SMI, structure model index; Tb.N, trabecular number; Tb.Pf, trabecular pattern factor; Tb.Th, trabecular thickness.

* Corresponding author: D. Singh, fax +91522 2771940, email divya_singh@cdri.res.in 
particularly in beans ${ }^{(12)}$. It is the major bioactive compound isolated from Astragalus membranaceus, which is a traditionally used medicinal herb for the treatment of diabetes ${ }^{(13)}$.

Previous studies demonstrated that formononetin, which is a structurally related methoxylated daidzein, is devoid of any uterine oestrogenicity and has osteoprotective effects. Studies in our laboratory showed that formononetin stimulated osteoblast differentiation via activation of the p38 mitogen-activated protein kinase pathway ${ }^{(14)}$. Daily oral administration of formononetin at $10.0 \mathrm{mg} / \mathrm{kg}$ per d dose to growing female Sprague-Dawley rats for 30 consecutive days increased the bone mineral density at various anatomic positions studied ${ }^{(15)}$. Formononetin was also shown to have a substantial bone anabolic effect in osteopenic adult Sprague-Dawley rats when administered for 12 weeks daily using the oral route. Formononetin had no oestrogenic or antioestrogenic effect as assessed by uterine wet weight. Formononetin treatment did not result in hypertrophy of luminal and glandular epithelium as observed using $17 \beta-\mathrm{E} 2^{(15)}$. As formononetin was devoid of any uterine oestrogenicity, we decided to study the boneregenerative capacity of formononetin in an ovariectomised (OVx) mouse model in which the cortical defect was generated in the femoral mid-diaphysis through drill-hole injury. We chose the Balb/c mouse model because it mimics postmenopausal osteoporosis and the fracture-related consequences associated with it ${ }^{(16,17)}$. Our study reveals that formononetin treatment repairs the cortical bone defect and enhances bone regeneration in OVx osteopenic mice. As bone regeneration is accompanied by increased expression of osteogenic markers like $B M P 2$, Runx2 and osteocalcin $(O C N)^{(18,19)}$, we also demonstrate enhanced recruitment of osteoprogenitors at the injury site.

\section{Methods}

\section{Reagents and chemicals}

Cell-culture media, supplements and calcein were acquired from Sigma-Aldrich. Antibodies for immunofluorescence were obtained from Cell Signaling Technology and Santa Cruz Biotechnology. Human PTH (1-34) was acquired from Calbiochem.

\section{Synthesis of formononetin}

A mixture of $p$-methoxy phenyl acetic acid (9.3 g, $0.056 \mathrm{~mol}$ ) in boron trifluoride diethyl etherate $(20 \mathrm{ml})$ and resorcinol $(5.5 \mathrm{~g}, 0.050 \mathrm{~mol})$ was heated at $100^{\circ} \mathrm{C}$ for $8 \mathrm{~h}$. The reaction mixture was poured into water, extracted with ethyl acetate and dried over anhydrous sodium sulphate. After filtration, the solvent was removed under vacuum at $40-45^{\circ} \mathrm{C}$ to yield the yellowish crude product. The product was suspended in dimethylformamide $(90 \mathrm{ml})$ and then boron trifluoride etherate $(20 \mathrm{ml})$ and methane sulphonyl chloride $(8 \mathrm{ml})$ were carefully added. The red solution was heated at $80^{\circ} \mathrm{C}$ for $3 \mathrm{~h}$ and then poured onto ice. The off-white precipitate was filtered and purified by crystallisation in ethanol (online Supplementary Fig. S1(a)). The purity of formononetin was analysed using HPLC with a C-18 reverse-phase column $(4.6 \times 250 \mathrm{~mm})$; the mesh size was $5 \mu \mathrm{m}$ and a gradient solution of acetonitrile:water was used as an eluent with a flow rate of $0.8 \mathrm{ml} / \mathrm{min}$ for $25 \mathrm{~min}$.
The purity of formononetin was analysed at $254 \mathrm{~nm}$ and found to be $99.57 \%$ (online Supplementary Fig. S1(b)).

Physicochemical data are as follows. Yield: $82 \%$; mp: $256-258^{\circ} \mathrm{C}$; FTIR (KBr) $\nu_{\max }: 3420,2345,1600,1452,1389,1304,1230$, 1177, 1023 (per cm); ${ }^{1} \mathrm{H}-\mathrm{NMR}$ ( $400 \mathrm{MHz}$, DMSO- $\left.d_{6}\right) \delta$ (ppm): $8.29(\mathrm{~s}, 1 \mathrm{H}), 7.96(\mathrm{~d}, J=8.9 \mathrm{~Hz}, 1 \mathrm{H}), 7.48(\mathrm{dd}, J=2 \cdot 1,8.9 \mathrm{~Hz}, 2 \mathrm{H})$, $6.97(\mathrm{dd}, J=2 \cdot 1,8 \cdot 8 \mathrm{~Hz}, 2 \mathrm{H}), 6.94(\mathrm{dd}, J=2 \cdot 1,8.6 \mathrm{~Hz}, 1 \mathrm{H})$, $6.87(\mathrm{~d}, J=2.1 \mathrm{~Hz}, 1 \mathrm{H}), 3.77 \quad(\mathrm{~s}, 3 \mathrm{H}) ;{ }^{13} \mathrm{C}-\mathrm{NMR} \quad(100 \mathrm{MHz}$, DMSO- $\left.d_{6}\right) \delta(\mathrm{ppm}): 175 \cdot 1,163 \cdot 0,159 \cdot 4,157 \cdot 9,153 \cdot 5,130 \cdot 5,127 \cdot 7$, $124 \cdot 7,123 \cdot 6,117 \cdot 1,115 \cdot 6,114 \cdot 0,102 \cdot 6,55 \cdot 6$; ESI-MS: $[\mathrm{M}+\mathrm{H}]^{+}=269$ (online Supplementary Fig. S2(a) and (b)).

\section{Animals and experimental procedures}

The study was conducted in accordance with the current legislation for animal experiments (Institutional Animal Ethical Committee at Central Drug Research Institute, Lucknow Institute (CPSCEA registration no. 34/1999, dated 3 November 1999, extended to 2016, approval reference no. IAEC/2012/71 N/Renew-4(30/16)/dated February 12, 2016) received from the National Laboratory Animal Centre, Council of Scientific and Industrial Research-Central Drug Research Institute (CDRI). All mice were housed in individual cages at $25^{\circ} \mathrm{C}$, in $12 \mathrm{~h}$ light $-12 \mathrm{~h}$ dark cycles. Overall, eighty adult Balb/c mice (8-10 weeks old; weighing 25 (SEM 5) g each) were randomly divided into eight equal groups (ten mice/group) as follows: sham operated (ovary intact) + vehicle (gum acacia in distilled water p.o.), $10 \mathrm{~d}$; OVx + vehicle, $10 \mathrm{~d} ;$ OVx + formononetin $(10.0 \mathrm{mg} / \mathrm{kg}$ per $\mathrm{d})$; $10 \mathrm{~d} ; \mathrm{OVx}+\mathrm{PTH}(10 \cdot 0 \mu \mathrm{g} / \mathrm{kg}$ per d), $10 \mathrm{~d}$; sham operated (ovary intact) + vehicle (gum acacia in distilled water p.o.), $21 \mathrm{~d}$; OVx + vehicle, $21 \mathrm{~d} ;$ OVx + formononetin $(10.0 \mathrm{mg} / \mathrm{kg}$ per d), $21 \mathrm{~d}$; and OVx + PTH $(10 \cdot 0 \mu \mathrm{g} / \mathrm{kg}$ per d), $21 \mathrm{~d}$. A total of ten mice per group were selected for the study; whereas formononetin and gum acacia were administered daily by oral gavage, PTH was subcutaneously administered. For the study, animals were left for $30 \mathrm{~d}$ to develop osteopenia. In all, seven mice per group were selected for histomorphometric studies. The rest were used for RNA and immunohistochemical analysis.

\section{Drill-hole injury in the femur}

A drill-hole injury was created in the sham-operated and formononetin-treated groups as described earlier ${ }^{(20)}$. Under anaesthetic conditions (ketamine-xylazine, $80-120 \mathrm{mg} / \mathrm{kg}$ : $10-16 \mathrm{mg} / \mathrm{kg}$, intramuscular), a mid-diaphyseal fracture was generated in the midfemur by a straight incision of the front skin. Choice of ketamine and xylazine as surgical anaesthetics was on the basis of their muscle-relaxant properties and ability to induce sedation and immobility. The periosteum was removed to expose the femoral bone surface. A drill-hole injury of $0.6 \mathrm{~mm}$ was created in the mid-diaphysis of the femur. Treatment started from the day after drill-hole creation. Formononetin at doses of $10.0 \mathrm{mg} / \mathrm{kg}$ were administered orally for 10 and $21 \mathrm{~d}$. PTH $(10 \cdot 0 \mu \mathrm{g} / \mathrm{kg}$ per d) was administered as a reference standard. The fluorochrome calcein $(20 \mathrm{mg} / \mathrm{kg})$, dissolved in normal saline, was given to all animals via the intraperitoneal route 24-h before autopsy. Mice were killed under anaesthesia and femur bones in which the drill-hole injury was created were placed in $70 \%$ isopropanol for fixation 
and later subjected to microarchitecture analysis. For the assessment of bone microarchitectural parameters strictly at the drill-hole site, bones were fixed in an acrylic material and $50 \mu \mathrm{m}$ sections were trimmed using bone cutter (IsoMet). To photograph calcein binding, a confocal microscope was used (LSM510 Meta; Carl Zeiss) with the aid of appropriate filters. Calcein-binding intensity at the drill site as an indication of the amount of bone regenerated was calculated using Carl Ziess AM4.2 image-analysis software.

\section{Microcomputed tomography}

Microcomputed tomography $(\mu \mathrm{CT})$ of excised bones was performed using the 1076 CT scanner (SkyScan) according to a previously published protocol ${ }^{(21)}$. Bones were cleaned of muscles and soft tissues and subjected to an X-ray source of $70 \mathrm{kV}, 100 \mathrm{~mA}$ with a pixel size of $9 \mu \mathrm{m}$ for scanning. Reconstruction of images was performed using SkyScan Nrecon software, which helps network-distributed reconstruction carried out on four personal computers running simultaneously. The callus region of the bone was captured by drawing an ellipsoid contour using CT analyser software. Microarchitectural parameters encompassing bone-volume fraction (BV/TV), trabecular thickness (Tb.Th), trabecular number (Tb.N), trabecular pattern factor (Tb.Pf), structure model index (SMI) and degree of anisotropy (DA) were assessed as described earlier ${ }^{(19,21)}$.

\section{Histology}

Femurs were detached by disarticulation of the forelimb through the knee joint. After removal of skin and superficial layers of soft tissues, the femurs encompassing the drill-hole site were fixed in $4 \%$ paraformaldehyde in PBS, decalcified in EDTA, followed by embedding for sectioning and staining with haematoxylin-eosin.

\section{Quantitative real-time PCR}

For gene-expression studies, the portion surrounding the drill hole was dissected at a 1-mm margin from the femoral bone. Tissues were carefully harvested and cleaned of muscle and soft connective tissues. The specimen was frozen in $\mathrm{N}_{2}$ and crushed into powder. Total RNA was extracted using Trizol. Complementary DNA (cDNA) was synthesised from $1 \mu \mathrm{g}$ of total RNA using RevertAidkit (Fermentas). SYBR green chemistry was used for quantitative assessment of mRNA for runt-related transcription factor 2 (RUNX2), OCN and BMP2. The design of sense and antisense oligonucleotide primers was based on published cDNA sequences using the Universal ProbeLibrary (Roche Diagnostics). Primer sequences are mentioned in Table 1. For real-time PCR, cDNA was amplified in LightCycler 480 (Roche Diagnostics). $\beta$-actin was used as the normalising gene.

\section{Immunofluorescence}

Femurs with drill-hole injury were dissected free of connective tissues, fixed in $4 \%$ buffered formalin, decalcified in $1 \%$ EDTA and embedded in paraffin. Transverse sections of $5 \mu \mathrm{m}$ were then cut from each sample. Sections were initially deparaffinised using xylene, rehydrated through an ethanol gradient and
Table 1. Primer sequences used for quantitative real-time PCR

\begin{tabular}{ll}
\hline Gene names & Primer sequence \\
\hline Runt-related transcription factor 2 & F: CCCGGGAACCAAGAAATC \\
& R: CAGATAGGAGGGGTAAGACTGG \\
Osteocalcin & F: TGAGGACCATCTTCTGCTCA \\
BMP2 & F: AGGATCTGTACCGCAGGCACT \\
$\beta$-Actin & R: GGTGATGGCCAAGACTAAGG \\
& F: GGAGGGGGTTGAGGTGTT \\
& R: GTGTGCACTTTTATTGGTCTCAA \\
\hline
\end{tabular}

BMP2, bone morphogenetic protein 2 .

permeabilised with $0 \cdot 1 \%$ Triton X-100 followed by blocking with $1 \%$ bovine serum albumin (BSA). These were then incubated with primary antibodies for RUNX2 and OCN diluted in 1\% BSA (1:100) at $4^{\circ} \mathrm{C}$ overnight. Sections were again washed with PBS and incubated with fluorescent Alexa Fluor-488 goat anti-rabbit IgG $(\mathrm{H}+\mathrm{L})$ (1:500 dilution in PBS) (Molecular Probes) at room temperature for $1 \mathrm{~h}$. Sections were counterstained with 4,6-diamidino-2-phenylindole (DAPI) for $15 \mathrm{~min}$ in dark. After the 15min incubation, slides were washed with PBS and mounted using Vectashield (Vector Laboratories). Sections were visualised under EVOS FL Imaging System (Life Technologies).

\section{Statistical analysis}

Data are expressed as means with their standard errors. The data obtained in experiments with multiple treatments were subjected to one-way ANOVA followed by the NewmanKeuls test for significance using Prism version 3.0 software. Qualitative observations have been represented following assessments made by three individuals blinded to the experimental designs.

\section{Results}

Effect of formononetin on bone healing at day 10 post injury

At day 10 animals were autopsied and femur bones were isolated. The pattern of bone healing in the immature trabeculae of woven bones was determined at days 10 and 21 post injury using $\mu \mathrm{CT}$ analysis. Representative three-dimensional (3D) images at $10 \mathrm{~d}$ post fracture are shown in Fig. 1(a). BV/TV and Tb.N were significantly reduced in OVx mice compared with the sham group $(P<0 \cdot 001)$. Treatment of OVx mice with PTH led to a significant increase in BV/TV $(P<0.001)$ and Tb.N $(P<0.05)$. However, at $10 \mathrm{~d}$, formononetin treatment exhibited no significant increase in BV/TV and Tb.N compared with OVx (Fig. 1(b)).

Tb.Th, as expected, was low in OVx animals compared with the sham group $(P<0 \cdot 001)$. Interestingly, treatment with formononetin significantly enhanced the Tb.Th in OVx animals compared with the OVx control group $(P<0 \cdot 01)$. PTH treatment of the OVx animals also significantly increased Tb.Th compared with OVx animals $(P<0 \cdot 01)$ (Fig. 1(b)).

Geometrical parameters like SMI, Tb.Pf and DA were higher in OVx animals compared with sham animals $(P<0 \cdot 001)$. Treatment 
(a)

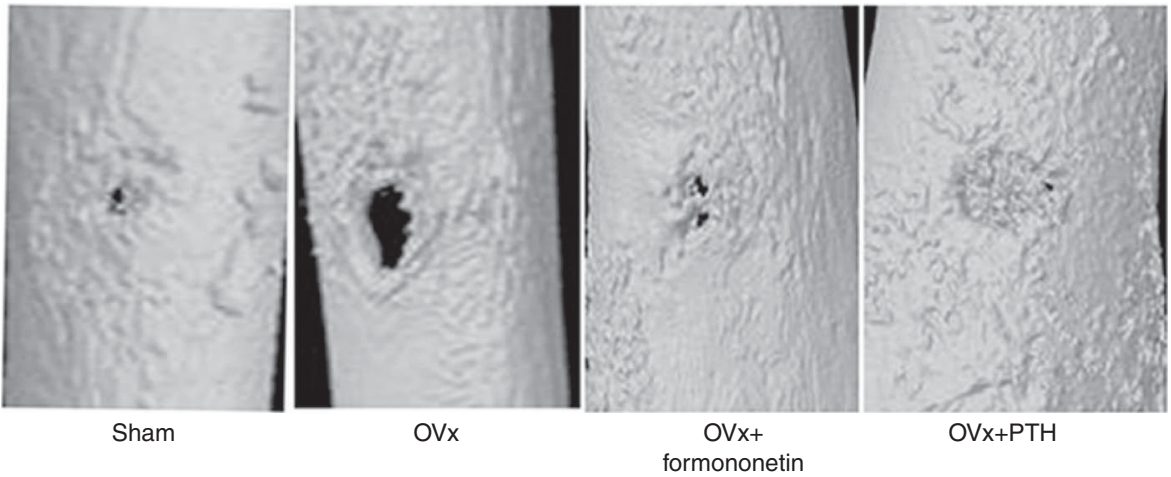

(b)

$\mathrm{BV} / \mathrm{TV}(\%)$
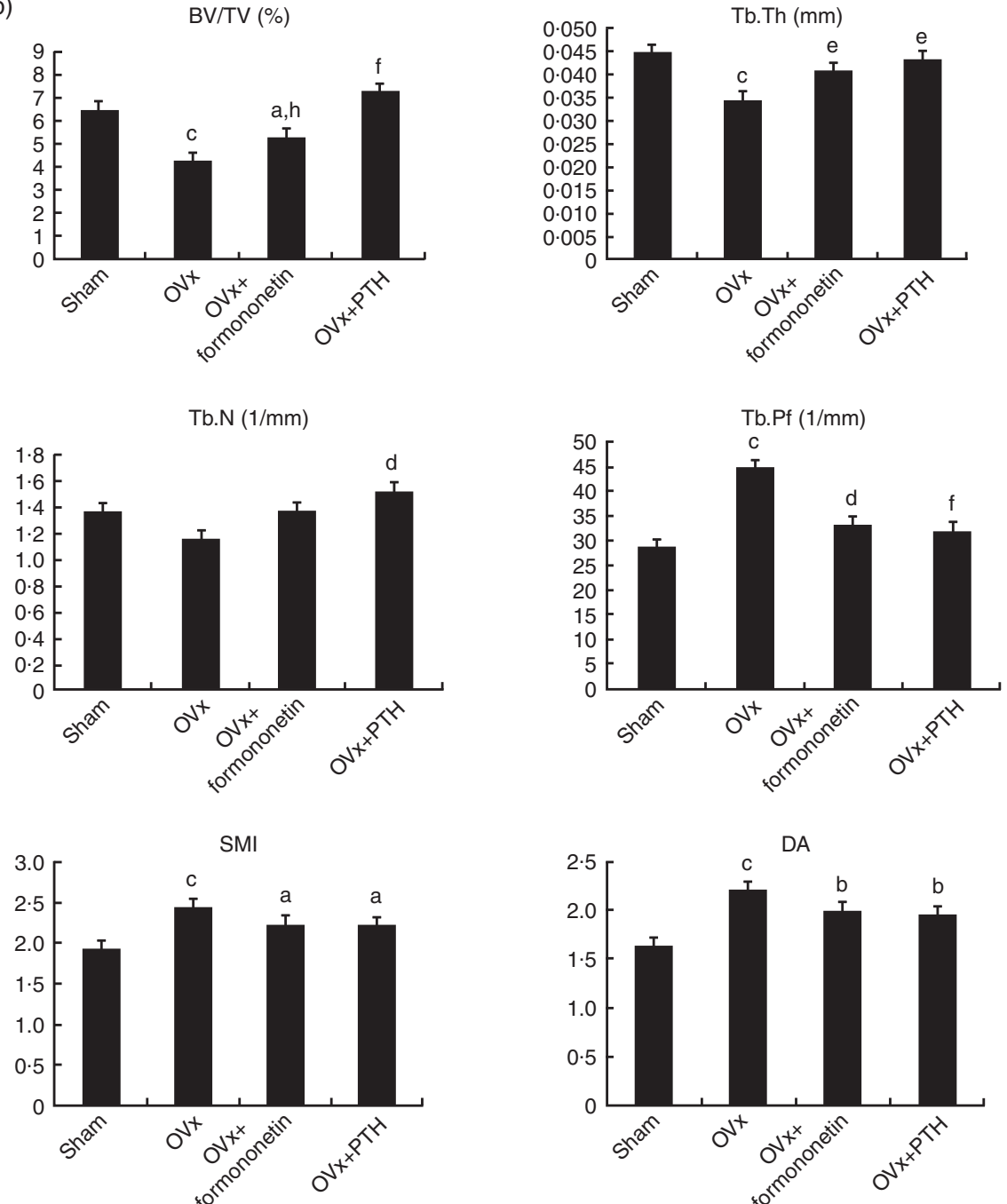

Fig. 1. Effect of formononetin on bone healing at day 10 post injury. (a) Representative three-dimensional microcomputed tomography images from the centre of the bony hole in various groups. (b) Quantitative presentation of microarchitectural parameters like trabecular bone volume (BV/TV), trabecular thickness (Tb.Th), trabecular network (Tb.N), trabecular pattern factor (Tb.Pf), structure model index (SMI) and degree of anisotropy (DA) in different groups. Values are means ( $n 10$ mice per group), with their standard errors represented by vertical bars and $95 \% \mathrm{Cl}$. Statistical analysis was performed using the non-parametric one-way ANOVA method followed by the Newman-Keuls test for significance using Prism version 3.0 software. OVx, ovariectomised; PTH, parathyroid hormone. ${ }^{a} P<0.05$ compared with the sham + vehicle group; ${ }^{b} P<0.01$ compared with the sham + vehicle group; ${ }^{c} P<0.001$ compared with the sham + vehicle group; ${ }^{d} P<0.05$ compared with the OVx group; ${ }^{\mathrm{e}} P<0.01$ compared with the OVx group; ${ }^{\mathrm{f}} P<0.001$ compared with the $\mathrm{OVx}$ group; ${ }^{\mathrm{h}} P<0.01$ compared with the $\mathrm{OVx}+$ formononetin group. 
with formononetin significantly reduced Tb.Pf $(P<0 \cdot 001)$ compared with the OVx control group; however, both SMI and DA were unchanged by formononetin treatment (Fig. 1(b)). Similar results were obtained with PTH. Thus, at $10 \mathrm{~d}$ the effect of formononetin is not especially profound.

\section{Effect of formononetin on bone healing at day 21 post injury}

Representative 3D images at $21 \mathrm{~d}$ post fracture are shown in Fig. 2(a). At $21 \mathrm{~d}, \mathrm{BV} / \mathrm{TV}$ in OVx animals was significantly lower than that in the sham group $(P<0 \cdot 001)$. OVx animals treated with formononetin exhibited a significant increase in BV/TV compared with the OVx control group $(P<0.01)$, almost equal to sham, but less than $\mathrm{OVx}+\mathrm{PTH}$-treated groups $(P<0.05)$ (Fig. 2(b)). Tb.Th was also decreased in the OVx group compared with the sham group $(P<0.001)$ at $21 \mathrm{~d}$. Treatment of $\mathrm{OVx}$ mice with formononetin led to increased $\mathrm{Tb}$.Th compared with the $\mathrm{OVx}$ control group $(P<0.001)$ (Fig. 2(b)). The increase produced by formononetin was similar to that in the PTH-treated group. Tb.N in the formononetin-treated group were also higher than those in the OVx control group $(P<0 \cdot 001)$ (Fig. 2(b)). Interestingly, the increment observed in Tb.N after formononetin treatment of OVx animals was even greater than the sham group $(P<0.05)$ and similar to the PTH group (Fig. 2(b)).

Geometrical parameters like Tb.Pf, SMI and DA were significantly higher in OVx animals in comparison with the sham group. Treatment with formononetin, however, reduced these parameters $(P<0.001)$ and brought them equivalent to the sham and PTH-treated groups (Fig. 2(b)). Thus, at $21 \mathrm{~d}$, formononetin was effective in promoting bone healing at the injury site.

\section{Formononetin promotes bone regeneration}

The effect of formononetin was studied on bone regeneration at the drill-hole site in OVx mice on days 10 and 21. The histological appearance of the callus suggested that compared with the OVx control group, formononetin treatment promotes accelerated tissue formation with potentially greater extracellular matrix deposition in a day-dependent manner, with $21 \mathrm{~d}$ exhibiting better extracellular deposition. The effect observed with PTH was the same as that observed with formononetin (Fig. 3(a)).

The effect of formononetin on the regeneration of bone was quantified on the basis of the calcein label (mineral deposition) at the site of the drill hole on days 10 and 21. At $10 \mathrm{~d}$, it was observed that OVx mice treated with formononetin at a dose of $10 \cdot 0 \mathrm{mg} / \mathrm{kg}$ have increased mineral deposition (measured from the intensity of calcein labelling in the drill hole) compared with the OVx control group $(P<0.001)$. The effect observed with PTH treatment in the OVx animals, though, was better than that in the formononetintreated OVx group $(P<0.05)$ (Fig. 3(b)).

Even at $21 \mathrm{~d}$, formononetin-treated OVx mice exhibited increased mineral deposition $(P<0 \cdot 001)$. However, it is important to note that the increase in mineral deposition observed with the formononetin treatment was significantly better than that in PTH-treated OVx animals $(P<0.05)$ and the sham group $(P<0.001$ (Fig. 3(b)). Thus, formononetin promotes bone regeneration and has the same efficacy as PTH in the healing of drill-hole injury.

\section{Expression of osteogenic markers at the site of bone} regeneration

Callus tissues were isolated from the region adjoining the injury area in various treated groups at days 10 and 21. RNA was isolated from these tissues to measure the expression of osteogenic gene markers.

RUNX2, OCN and BMP2 expression levels were significantly lower in calluses from OVx mice at $10 \mathrm{~d}$. Treatment with formononetin in OVx mice led to significant enhancement in RUNX2 $(P<0 \cdot 01), O C N(P<0 \cdot 01)$ and BMP2 transcript levels $(P<0 \cdot 001)$ (Fig. 4(a)). RUNX2 and OCN levels in PTH-treated groups were significantly higher than in formononetin-treated groups $(P<0 \cdot 01)$. However, BMP2 transcript levels were significantly higher in the formononetin-treated group compared with the PTH group $(P<0.001)$ (Fig. 4(a)). Again, at $21 \mathrm{~d}$, increased transcript levels of RUNX2 $(P<0 \cdot 01), B M P 2(P<0 \cdot 01)$ and $O C N(P<0.00)$ were observed in formononetin-treated groups (Fig. 4(b)). BMP2 transcript levels were higher in the formononetin-treated group compared with the PTH-treated OVx group (Fig. 4(b)).

\section{Immunofluorescence localisation of osteocalcin and runt-related transcription factor 2 at the injury site}

In order to confirm if rapid bone healing in formononetin-treated OVx mice is attributable to the increased recruitment of osteoprogenitors at the injury site, immunohistochemical localisation of RUNX2 and OCN at the injury site was carried out in different groups. Immunohistochemical localisation of RUNX2 and $O C N$ at the injury site demonstrated predominant localisation of RUNX2 and $O C N$ in formononetin-treated OVx mice compared with the OVx control (Figs. 5 and 6). Immunofluorescence analysis revealed an intense staining for OCN and RUNX2 (anti-OCN and anti-RUNX2 antibody showing green staining) at the injury site in formononetin-treated groups at days 10 and 21, with a higher intensity at the 21-d time point. DAPI was used as a nuclear counterstain (blue staining). OCN and RUNX2, when merged with DAPI, demonstrated a visually merged image (Figs. 5 and 6).

\section{Discussion}

In this study, we determine the role of formononetin in bone healing and regeneration. In previous reports, we had demonstrated the osteonabolic activity of formononetin ${ }^{(15)}$. Besides, it was devoid of any uterine oestrogenicity that otherwise increases the risk for endometrial carcinoma. The current study was carried out in a cortical bone defect model in which a drill-hole injury of $0.6 \mathrm{~mm}$ was generated in the mid-diaphysis of the femur. The characteristics of bone healing were intramembranous dominant with low endochondral ossification. For the study, PTH was taken as the positive reference because of its proven fracture-healing effect $^{(22)}$. After the injury, treatments were administered for a period of $21 \mathrm{~d}$ and bone samples were collected at different time points of 10 and $21 \mathrm{~d}$ for microarchitectural analysis of woven regenerating bone as it is synthesised initially at the defect area and replaced later on with lamellar bone ${ }^{(23)}$. At day 10 post injury, formononetin treatment did not result in increased BV/TV 
(a)

(21d)

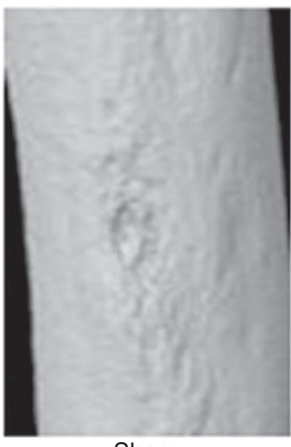

Sham

(b)
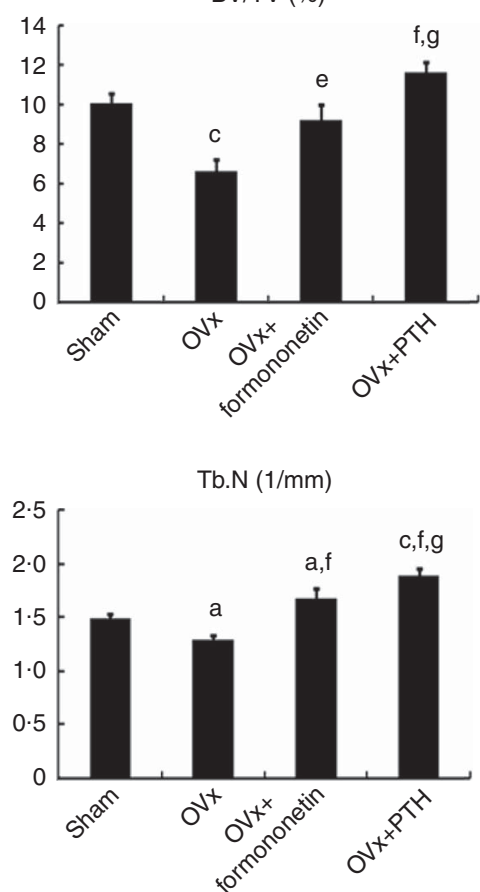

SMI

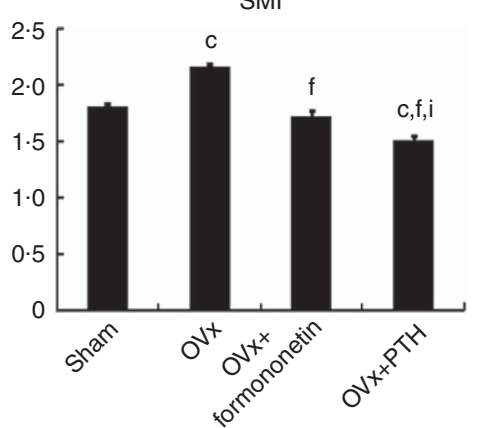

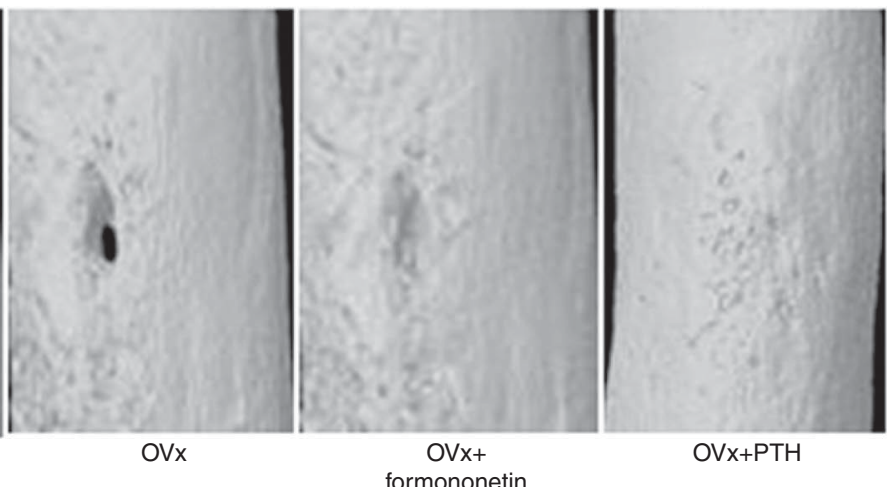

formononetin
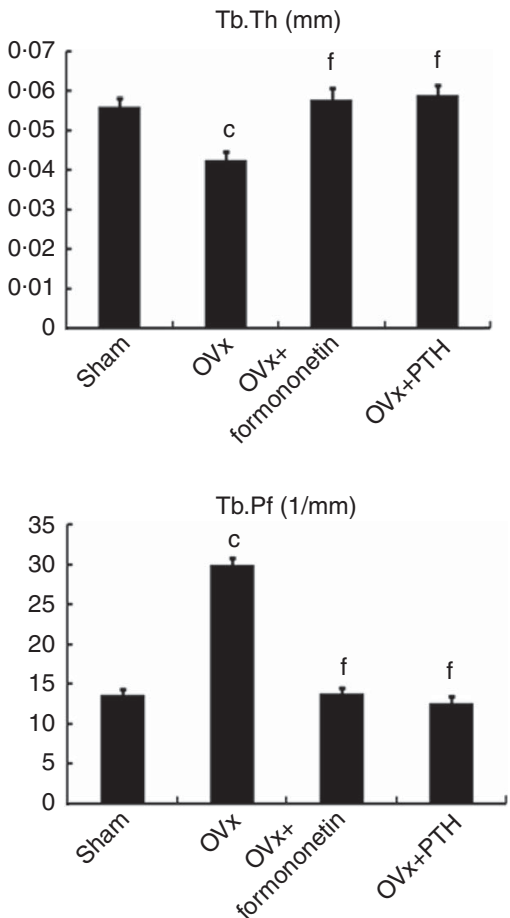

DA

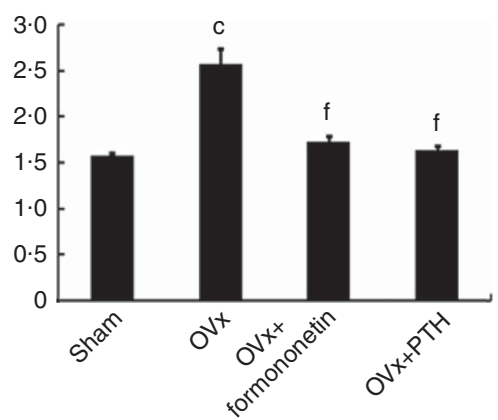

Fig. 2. Effect of formononetin on bone healing at day 21 post injury. (a) Representative three-dimensional microcomputed tomography images from the centre of the bony hole in various groups. (b) Quantitative presentation of the microarchitectural parameters like trabecular bone volume (BV/TV), trabecular thickness (Tb.Th), trabecular network (Tb.N), trabecular pattern factor (Tb.Pf), structure model index (SMI) and degree of anisotropy (DA) in different groups. Values are means ( $n$ 10 mice per group), with their standard errors represented by vertical bars and $95 \% \mathrm{Cl}$. Statistical analysis was performed using the non-parametric one-way ANOVA method followed by the Newman-Keuls test for significance using Prism version 3.0 software. OVx, ovariectomised; PTH, parathyroid hormone. ${ }^{a} P<0.05$ compared with the sham + vehicle group; ${ }^{\mathrm{C}} P<0.001$ compared with the sham + vehicle group; ${ }^{\mathrm{e}} P<0.001$ compared with $\mathrm{OVx}$ group; ${ }^{\mathrm{f}} P<0.001$ compared with the OVx group; ${ }^{g} P<0.05$ compared with the $\mathrm{OVx}+$ formononetin group; ${ }^{i} P<0.001$ compared with the $\mathrm{OVx}+$ formononetin group. 
(a) $(10 \mathrm{~d})$

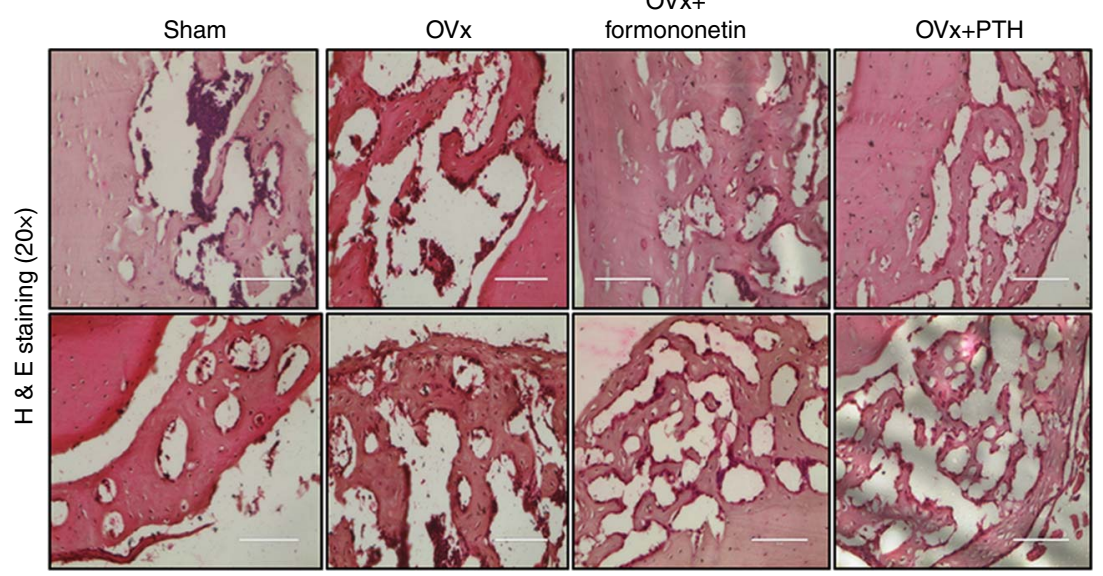

(b)

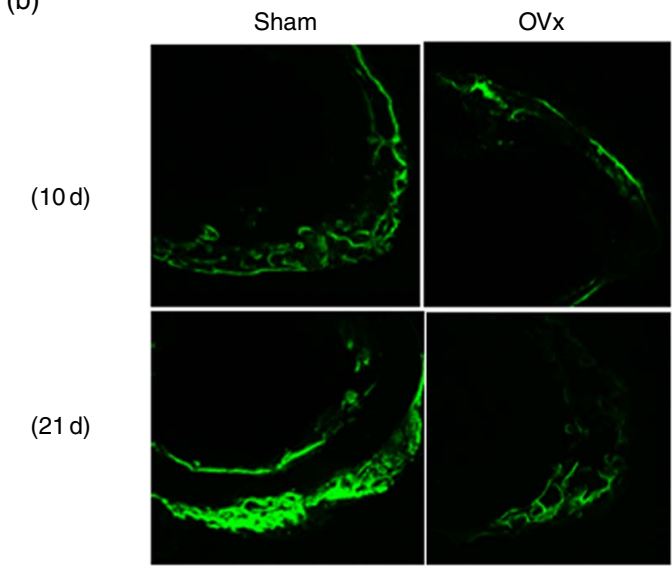

$\mathrm{OVx}+$

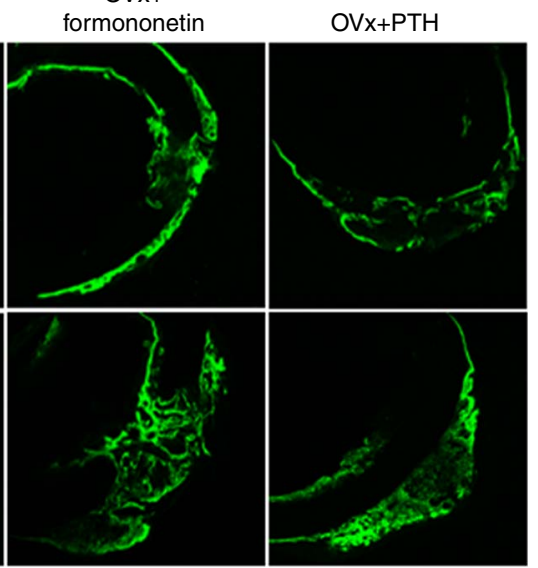

$(10 d)$

(21d)
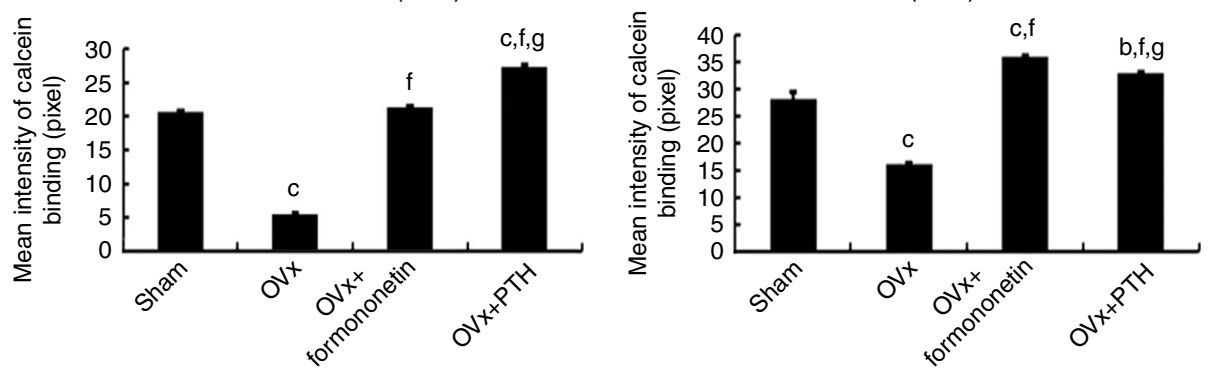

Fig. 3. Effect of formononetin on bone regeneration at the injury site. (a) Haematoxylin-eosin $(\mathrm{H} \& \mathrm{E})$ staining of newly regenerated bone at the injury sites of various groups at days 10 and 21. (b) Representative confocal images (10x) of calcein labelling shown in the drill holes of various groups at days 10 and 21 after injury and quantification of the mean intensity of the calcein label. Values are means ( $n 10$ mice per group), with their standard errors represented by vertical bars and $95 \% \mathrm{Cl}$. OVx, ovariectomised; PTH, parathyroid hormone. Statistical analysis was performed using the non-parametric one-way ANOVA method followed by the NewmanKeuls test for significance using Prism version 3.0 software. ${ }^{b} P<0.01$ compared with the sham + vehicle group; ${ }^{c} P<0.001$ compared with the sham + vehicle group; ${ }^{f} P<0.001$ compared with the $\mathrm{OVx}$ group; ${ }^{9} P<0.05$ compared with the $\mathrm{OVx}+$ formononetin group.

(reflecting a dense trabecular network) and Tb.N in comparison with the OVx control group. However, thinning of trabeculae at the injury site resulting from OVx was significantly overcome by formononetin. Similarly, indices that serve as a surrogate of biochemical strength like SMI and DA were unchanged in the formononetin-treated group but OVx-induced Tb.Pf was significantly decreased by formononetin treatment. Tb.Pf represents the contour of the trabecular surface, the ratio of convex:concave surfaces. Thus, larger Tb.Pf values in OVx mice indicate that the convex or the eroded contour is predominant. Our results indicate a decrease in Tb.Pf in the formononetin group in comparison with the OVx control group. PTH, which was used as a positive control, imparted much better effects at $10 \mathrm{~d}$.

At $21 \mathrm{~d}$, however, the bone healing observed in formononetintreated groups was significantly better than the OVx control group and also similar to the sham and PTH-treated group. Parameters like BV/TV, number and thickness were better presented in the formononetin group than in the OVx control group. Geometric 
(a)
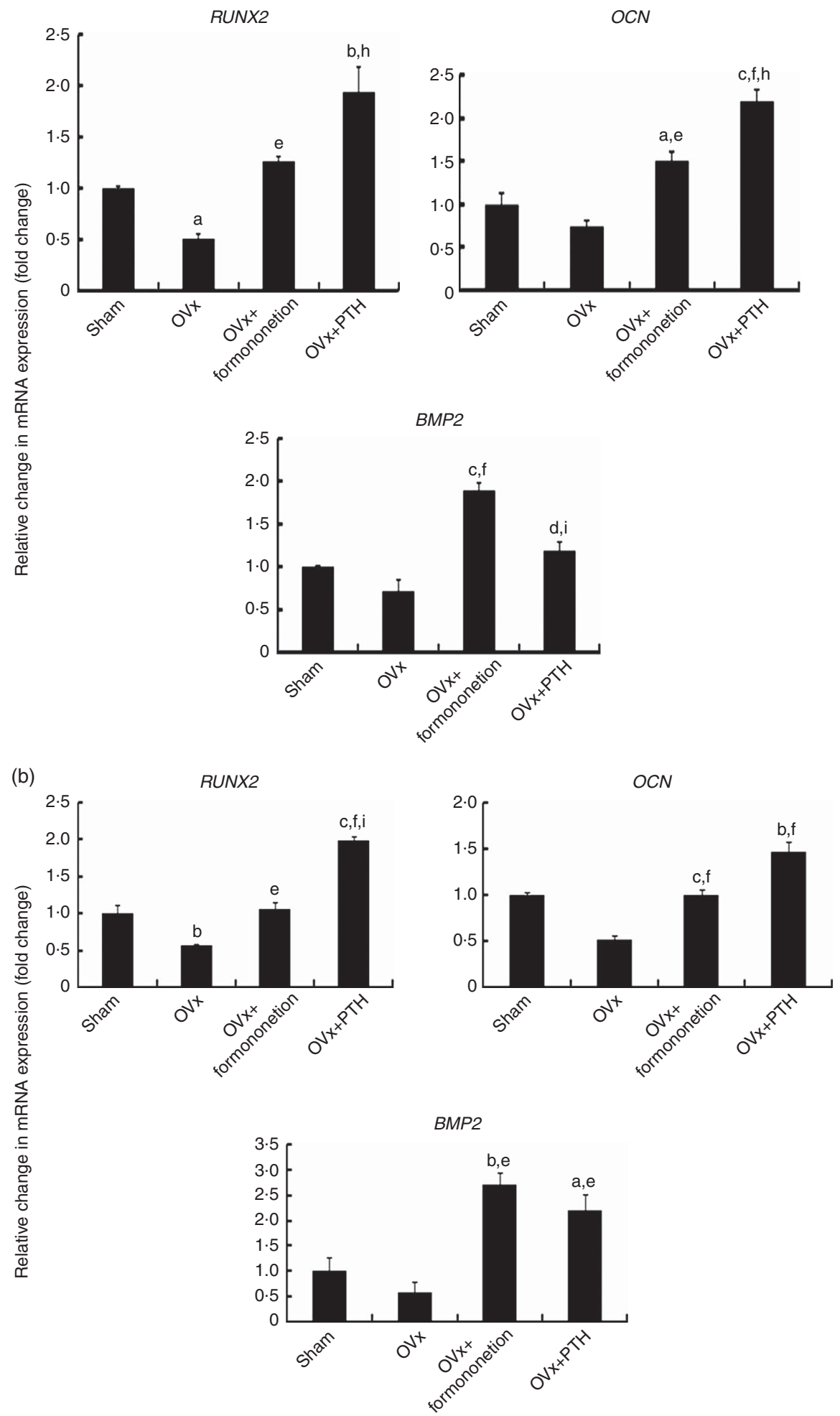

Fig. 4. Effect of formononetin on expression of osteogenic markers at days 10 (a) and 21 (b) post drill-hole injury. Data represent three independent experiments. Values are means ( $n 10$ mice per group), with their standard errors represented by vertical bars and $95 \% \mathrm{Cl}$. Statistical analysis was performed using the ANOVA method followed by the Newman-Keuls test for significance using Prism version 3.0 software. RUNX2, runt-related transcription factor 2; OCN, osteocalcin; OVx, ovariectomised; PTH, parathyroid hormone; $B M P 2$, bone morphogenetic protein. ${ }^{a} P<0.05$ compared with the sham + vehicle group; ${ }^{b} P<0.01$ compared with the sham + vehicle group; ${ }^{\mathrm{C}} P<0.001$ compared with the sham + vehicle group; ${ }^{\mathrm{d}} P<0.05$ compared with the OVx group; ${ }^{\mathrm{e}} P<0.01$ compared with the OVx group; ${ }^{f} P<0.001$ compared with the OVx group; ${ }^{\mathrm{h}} P<0.01$ compared with the $\mathrm{OVx}+$ formononetin group; ${ }^{\mathrm{i}} P<0.001$ compared with the $\mathrm{OVx}+$ formononetin group. 
(a)
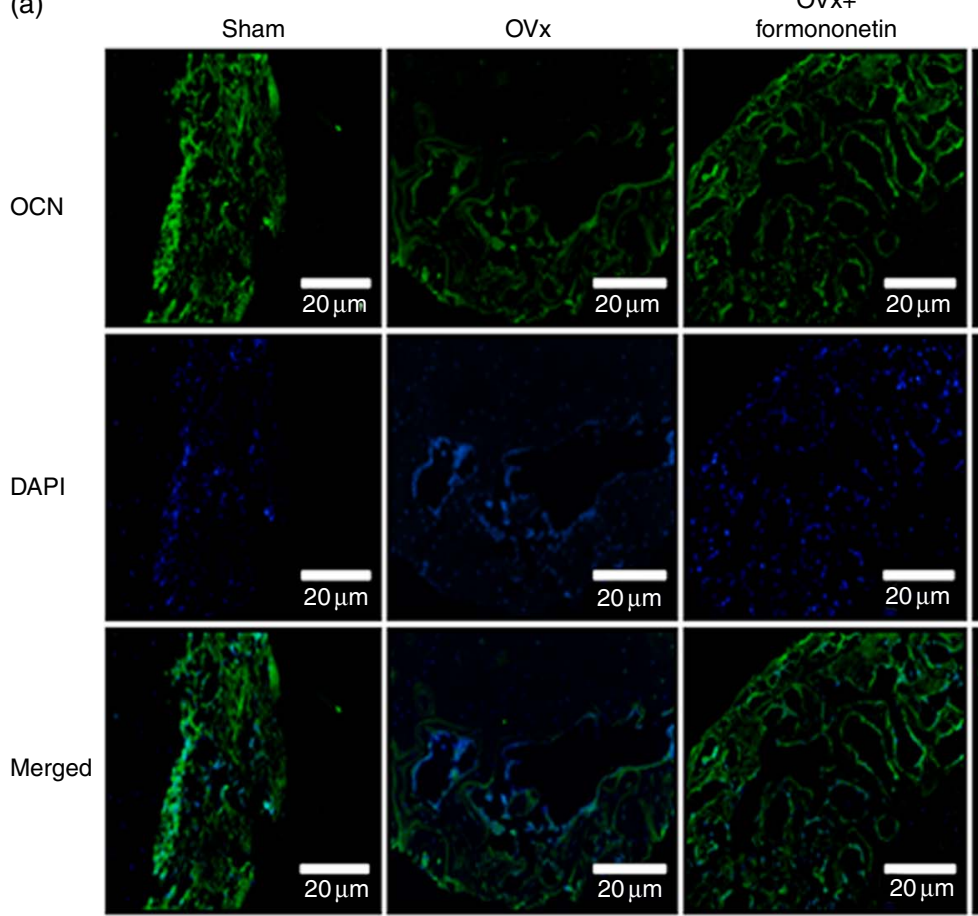

(b)

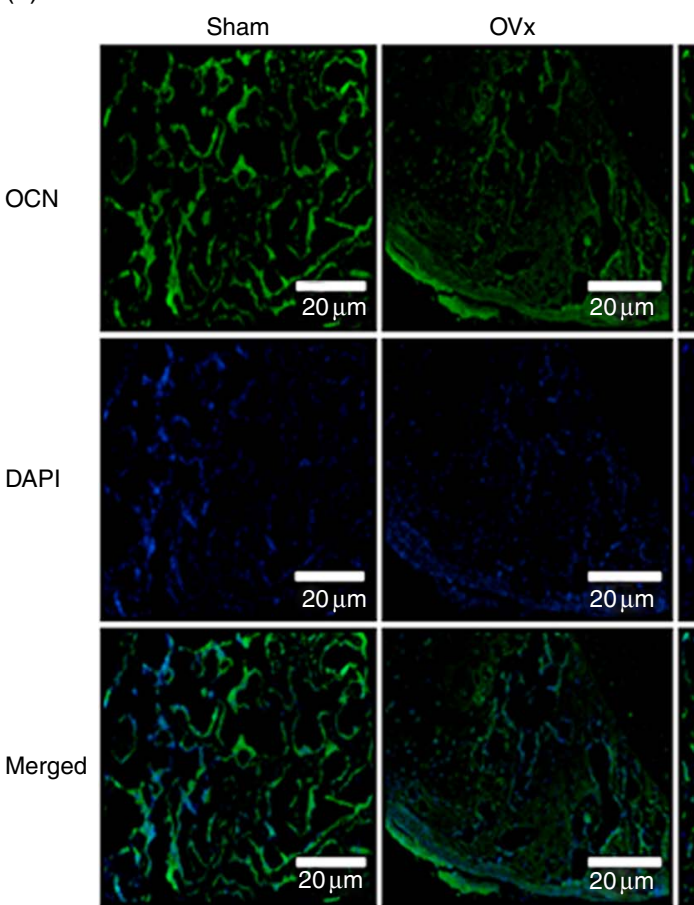

$\mathrm{OVx}+$ formononetin

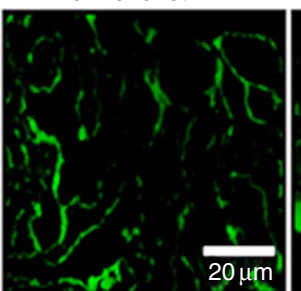
$\mathrm{OVx}+\mathrm{PTH}$
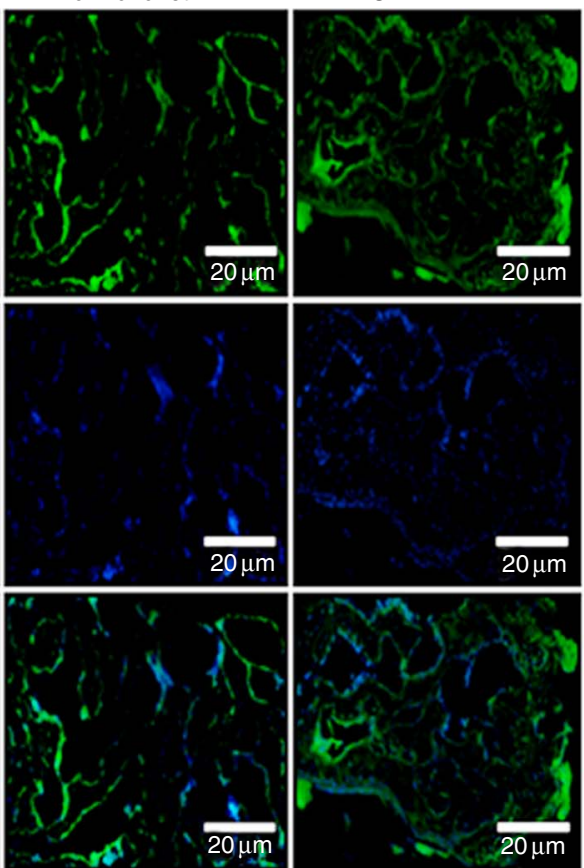

Fig. 5. Immunohistochemical localisation of osteocalcin $(O C M)$ at the injury site on days 10 (a) and 21 (b). Formononetin treatment led to the enhanced localisation of $O C N$ in the fracture callus compared with the sham group and was equivalent to the parathyroid hormone (PTH)-treatment group. Anti-OCN antibody shows green staining. 4',6-Diamidino-2-phenylindole (DAPI) was used as a nuclear counterstain (blue staining). OVx, ovariectomised.

indices like SMI, DA and Tb.Pf were also better presented. SMI distinguishes between rods and plates of bony trabeculae. SMI represents bone that consists purely of the plate or rod-like structures, respectively. Our results suggest a significant evolution of trabecular structures back from rod to plate form in OVx mice treated with formononetin. Thus, formononetin treatment of $\mathrm{OVx}$ mice significantly enhanced bone healing within the drill-hole region over a span of $21 \mathrm{~d}$ and the effect is equal to that of PTH. $\mu \mathrm{CT}$ data were corroborated by the labelling study of the fluorochrome calcein. It was observed that, compared with the sham-operated group, regeneration of bone in the drill hole was much reduced in OVx mice at days 10 and 21. 

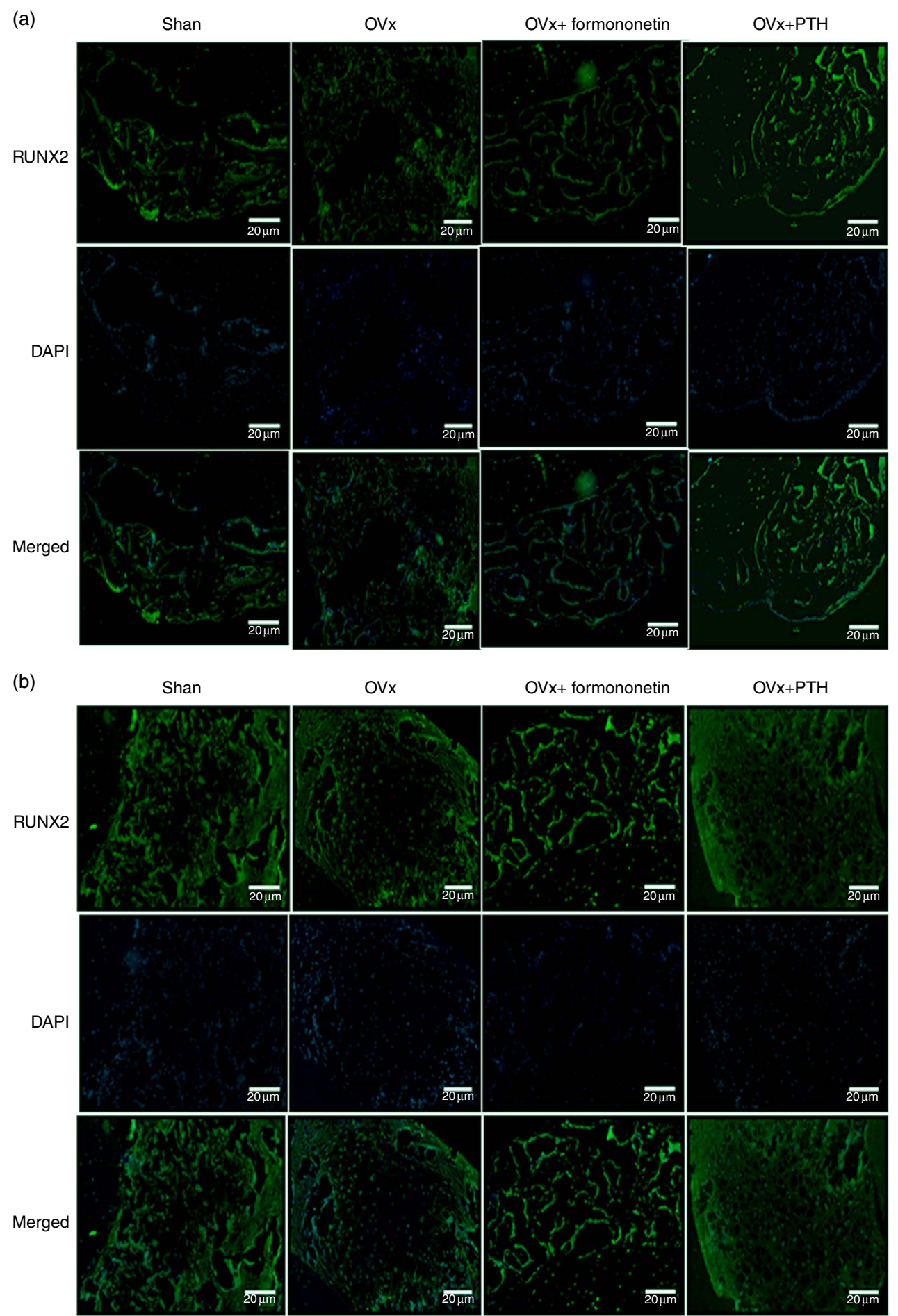

Fig. 6. Immunohistochemical localisation of runt-related transcription factor 2 (RUNX2) at the injury site on days 10 (a) and 21 (b). Formononetin treatment led to the enhanced localisation of RUNX2 in the fracture callus compared with the sham group and was equivalent to the parathyroid hormone (PTH)-treatment group. Anti-RUNX2 antibody shows green staining. 4',6-Diamidino-2-phenylindole (DAPI) was used as a nuclear counterstain (blue staining). OVx, ovariectomised.

However, treatment of OVx mice with formononetin significantly enhanced the process of filling up of newly generated bone in the drill hole and this healing effect was even better than sham and PTH groups at day 21. Histological analysis data also showed a larger area of regenerating bone in OVx animals treated with formononetin.
As our model was predominantly intramembranous, it was of interest to study whether formononetin treatment induced the recruitment of osteoprogenitors at the injury site. Transcript levels of osteogenic markers like RUNX2, OCN and BMP2 were determined in the tissues harvested from the region surrounding the defect site. Increased expression of these osteogenic markers 
was seen in formononetin-treated OVx animals. BMP2 mRNA, which has an important role in bone regeneration, was at even higher levels in the OVx animals treated with formononetin than in the PTH-treated groups. These observations were corroborated by enhanced immunolocalisation of RUNX2 and OCN at the site of injury in the formononetin-treated OVx animals.

Studies carried out to date suggest the lack of any notable side effects or toxic effects with formononetin treatment. A 90-d subchronic toxicity study by Li et al. ${ }^{(24)}$ on sodium formononetin30-sulphonate, which is a water-soluble derivative of formononetin, was evaluated in dogs at various doses. No mortality or abnormalities in body weight, clinical chemistry, haematology and histopathological examination were detected. Slight toxicity was observed at a $300 \mathrm{mg} / \mathrm{kg}$ dose but the compound was safe up to a $100 \mathrm{mg} / \mathrm{kg}$ dose. We tested our compound at $10 \mathrm{mg} / \mathrm{kg}$. The dog and human equivalent doses would be 1.5 and $0.8 \mathrm{mg} / \mathrm{kg}$, respectively, which is way below the $100 \mathrm{mg} / \mathrm{kg}$ level.

Overall, this study demonstrates the bone healing and bone regeneration potential of formononetin in a cortical bone defect model. This study also forms a strong case for the evaluation of formononetin in delayed-union and non-union fracture cases.

\section{Acknowledgements}

K. B. S. thanks the Indian Council of Medical Research, Government of India; M. D. thanks the Department of Biotechnology, Government of India for granting fellowships. The author K. D. is thankful to CSIR, New Delhi, India, for providing fellowship. R. M. is grateful to CSIR, New Delhi, India, for providing the emeritus scientist scheme with reference no. 21(1019)/16/EMR-II. The authors are also thankful to Sophisticated Analytical Instrument Facility (SAIF) division of CSIR-CDRI for providing spectral data. The authors thank Mr Geet K Nagar for helping with histology and Dr Kavita Singh for helping in the confocal microscopy facility of SAIF Division.

The research was supported by grants from the following: Centre for Research in Anabolic Skeletal Targets in Health and Illness (ASTHI), New Approaches towards Understanding of Disease Dynamics and to Accelerate Drug Discovery (UNDO), CSIR, Government of India.

D. S. and K. B. S. conceived and designed the experiments and analysed the data; K. B. S. carried out all experiments. M. D. and K. B. S. helped with the animal-related experiments. K. D. and R. M. have been involved in the synthesis of formononetin. D. S. wrote the manuscript.

The CDRI communication number is 9503 .

None of the authors has any conflicts of interest to declare.

\section{Supplementary material}

For supplementary material/s referred to in this article, please visit https://doi.org/10.1017/S0007114517001556

\section{References}

1. Claes L, Recknagel S \& Ignatius A (2012) Fracture healing under healthy and inflammatory conditions. Nat Rev Rheumatol 8, 133-143.

2. Ginaldi L, Di Benedetto MC \& De Martinis M (2005) Osteoporosis, inflammation and ageing. Immun Ageing $\mathbf{2}, 14$.
3. Zara JN, Siu RK, Zhang X, et al. (2011) High doses of bone morphogenetic protein 2 induce structurally abnormal bone and inflammation in vivo. Tissue Eng Part A 17, 1389-1399.

4. Kakar S, Einhorn TA, Vora S, et al. (2007) Enhanced chondrogenesis and Wnt signaling in PTH-treated fractures. J Bone Miner Res 22, 1903-1912.

5. Barnes GL, Kakar S, Vora S, et al. (2008) Stimulation of fracturehealing with systemic intermittent parathyroid hormone treatment. J Bone Joint Surg Am 90, Suppl. 1, 120-127.

6. Warden SJ, Komatsu DE, Rydberg J, et al. (2009) Recombinant human parathyroid hormone (PTH 1-34) and low-intensity pulsed ultrasound have contrasting additive effects during fracture healing. Bone 44, 485-494.

7. Sikon A \& Batur P (2010) Profile of teriparatide in the management of postmenopausal osteoporosis. Int $J$ Womens Health 2, 37-44.

8. Pietrogrande L (2009) Update on the efficacy, safety, and adherence to treatment of full length parathyroid hormone, PTH (1-84), in the treatment of postmenopausal osteoporosis. Int J Womens Health 1, 193-203.

9. Pandey R, Gautam AK, Bhargavan B, et al. (2010) Total extract and standardized fraction from the stem bark of Butea monosperma have osteoprotective action: evidence for the nonestrogenic osteogenic effect of the standardized fraction. Menopause 17, 602-610.

10. Maurya R, Yadav DK, Singh G, et al. (2009) Osteogenic activity of constituents from Butea monosperma. Bioorg Med Chem Lett 19, 610-613.

11. Medjakovic S \& Jungbauer A (2008) Red clover isoflavones biochanin $\mathrm{A}$ and formononetin are potent ligands of the human aryl hydrocarbon receptor. J Steroid Biochem Mol Biol 108, 171-177.

12. Saviranta NM, Julkunen-Tiitto R, Oksanen E, et al. (2010) Red clover (Trifolium pratense L.) isoflavones: root phenolic compounds affected by biotic and abiotic stress factors. J Sci Food Agric 90, 418-423.

13. Idris AI, Sophocleous A, Landao-Bassonga E, et al. (2008) Regulation of bone mass, osteoclast function, and ovariectomyinduced bone loss by the type 2 cannabinoid receptor. Endocrinology 149, 5619-5626.

14. Tyagi AM, Srivastava K, Singh AK, et al. (2012) Formononetin reverses established osteopenia in adult ovariectomized rats. Menopause 19, 856-863.

15. Gautam AK, Bhargavan B, Tyagi AM, et al. (2011) Differential effects of formononetin and cladrin on osteoblast function, peak bone mass achievement and bioavailability in rats. J Nutr Biochem 22, 318-327.

16. Sophocleous A \& Idris AI (2014) Rodent models of osteoporosis. Bonekey Rep 3, 614.

17. Dixit M, Singh KB, Prakash R, et al. (2017) Functional block of IL-17 cytokine promotes bone healing by augmenting FOXO1 and ATF 4 activity in cortical bone defect model. Osteoporos Int 28, 2207-2220.

18. Huang W, Yang S, Shao J, et al. (2007) Signaling and transcriptional regulation in osteoblast commitment and differentiation. Front Biosci 12, 3068-3092.

19. Dixit M, Raghuvanshi A, Gupta CP, et al. (2015) Medicarpin, a natural pterocarpan, heals cortical bone defect by activation of notch and wnt canonical signaling pathways. PLOS ONE 10, e0144541.

20. Sharan K, Mishra JS, Swarnkar G, et al. (2011) A novel quercetin analogue from a medicinal plant promotes peak bone mass achievement and bone healing after injury and exerts an anabolic effect on osteoporotic bone: the role of aryl hydrocarbon receptor as a mediator of osteogenic action. J Bone Miner Res 26, 2096-2111. 
21. Tyagi AM, Mansoori MN, Srivastava K, et al. (2014) Enhanced immunoprotective effects by anti-IL-17 antibody translates to improved skeletal parameters under estrogen deficiency compared with anti-RANKL and anti-TNF-alpha antibodies. J Bone Miner Res 29, 1981-1992.

22. Holzer G, Majeska RJ, Lundy MW, et al. (1999) Parathyroid hormone enhances fracture healing. A preliminary report. Clin Orthop Relat Res, 258-263.
23. Shapiro F (2008) Bone development and its relation to fracture repair. The role of mesenchymal osteoblasts and surface osteoblasts. Eur Cell Mater 15, 53-76.

24. Li C, Li G, Gao Y, et al. (2016) A 90-day subchronic toxicity study with sodium formononetin-3'-sulphonate (Sul-F) delivered to dogs via intravenous administration. Regul Toxicol Pharmacol 77, 87-92. 